\title{
Using Wet Electrospun PCL/Gelatin/CNT Yarns to Fabricate Textile-based Scaffolds for Vascular Tissue Engineering
}

\author{
Chen Jiang ${ }^{1,2}$, Kan Wang, ${ }^{2,}$, Yi Liu ${ }^{2,4}$, Chuck Zhang ${ }^{2,3}$, Ben Wang ${ }^{1,2,3}$ \\ ${ }^{1}$ School of Materials Science and Engineering, Georgia Institute of Technology, Atlanta, GA \\ 30332, USA \\ ${ }^{2}$ Georgia Tech Manufacturing Institute, Georgia Institute of Technology, Atlanta, GA 30332, \\ USA \\ ${ }^{3}$ H. Milton Stewart School of Industrial and System Engineering, Georgia Institute of \\ Technology, Atlanta, GA 30332, USA \\ ${ }^{4}$ School of Chemical and Biomolecular Engineering, Georgia Institute of Technology, Atlanta, \\ GA 30332, USA \\ *kan.wang@gatech.edu
}




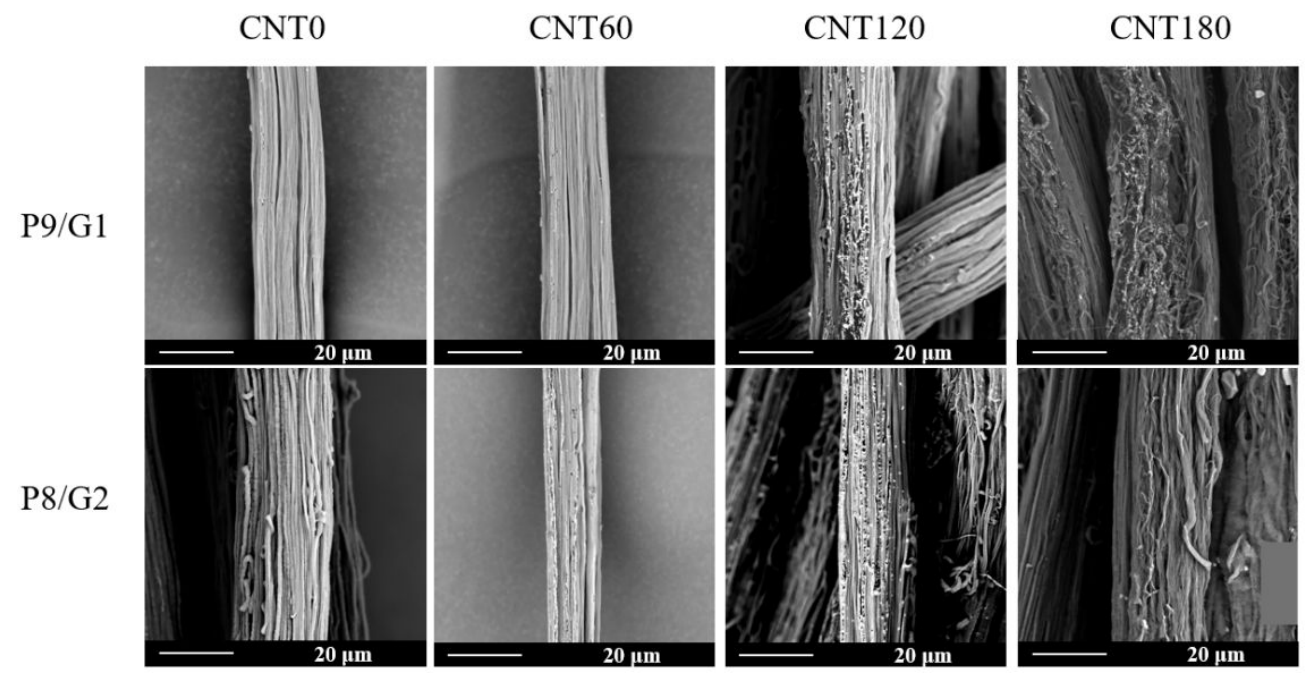

Figure S1. SEM images of wet electrospun bundles fabricated from different parameters. 

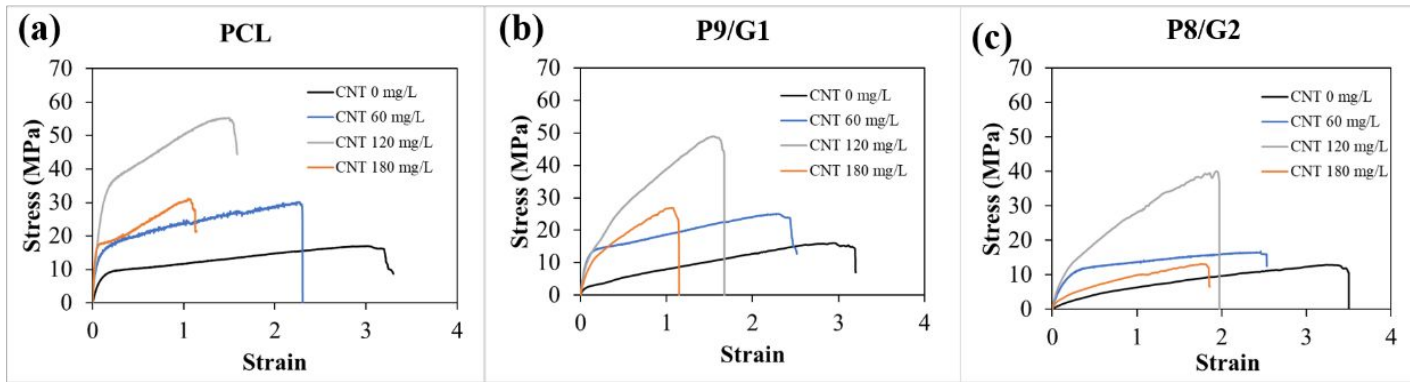

Figure S2. Representative stress-strain plots from the textile tests for wet electrospun yarns. 


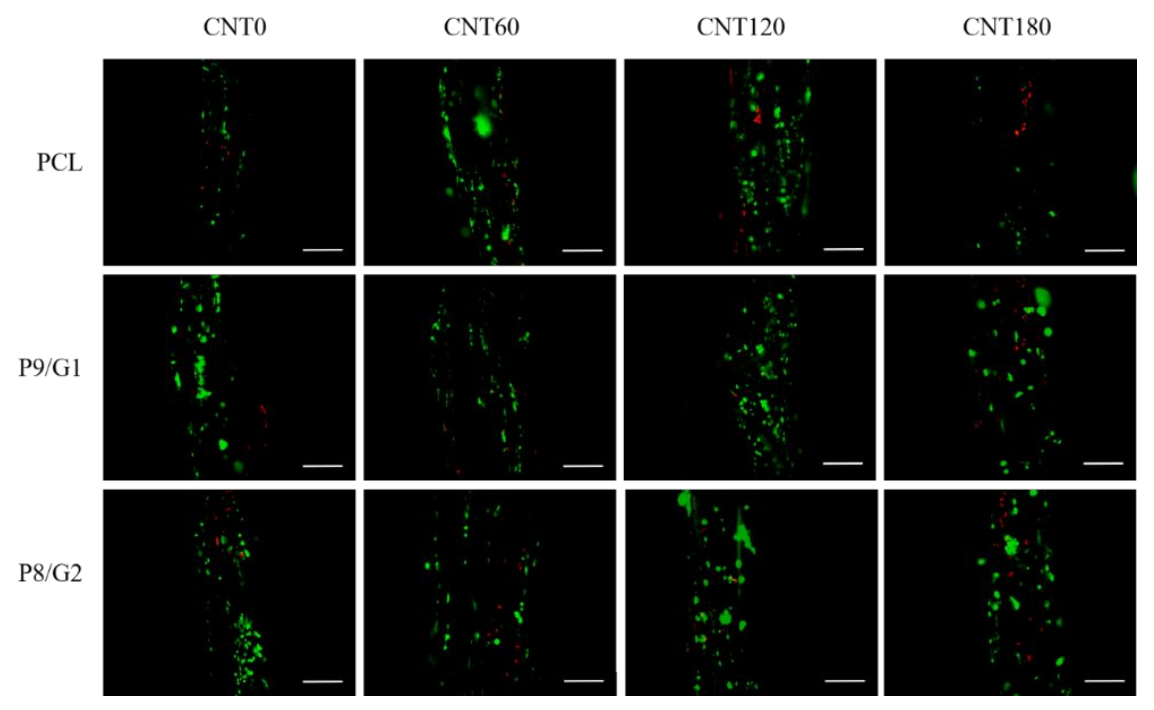

Figure S3. Fluorescent images of living cells (green) and dead cells (red) on wet electrospun yarns after cell cultivation for 2 days. (scale bar: $200 \mu \mathrm{m}$ ) 


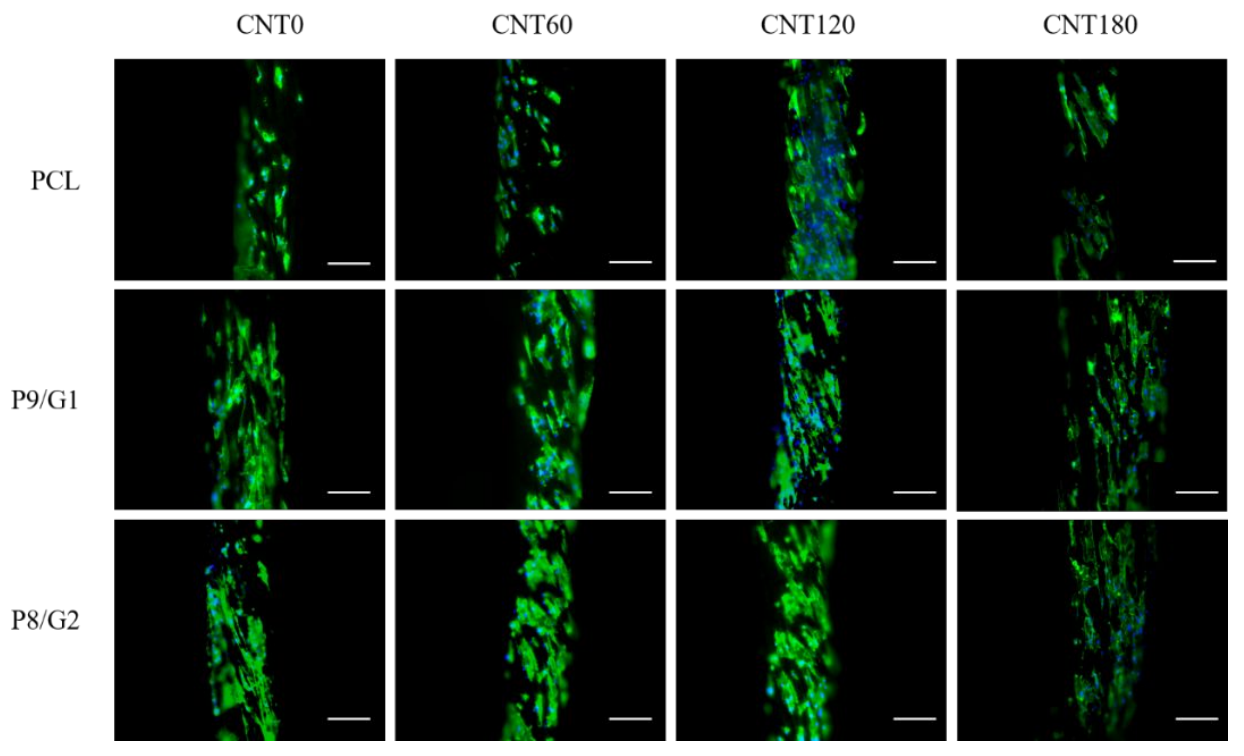

Figure S4. Fluorescent images of cells (green: Alexa Fluor, blue: DAPI) on wet electrospun yarns after cell cultivation for 2 days. (scale bar: $200 \mu \mathrm{m}$ ) 